\title{
The SIX survey: evolution and properties of AGN in the local universe
}

\author{
Eugenio Bottacini \\ Stanford University, USA \\ Email: eugenio.bottacini@stanford.edu
}

\begin{abstract}
Current most sensitive surveys at soft X-ray $(\sim 0.5-10 \mathrm{keV})$ energies by Chandra and XMM-Newton preferably sample AGN at high-redshift $(\mathrm{z} ; 0.5)$. At low-redshift $(\mathrm{z} ; 0.5)$, where AGN are supposed to be in their evolution end-stage, these sources are very sparsely sampled. The low-redshift universe is best fathomed at hard $\mathrm{X}$-ray energies (i.15 keV) by the INTEGRAL and the Swift missions with their coded-mask telescopes IBIS/ISGRI and BAT respectively. These instruments have two major advantages: 1) they have a huge field of view, hence allowing to sample a large number of AGN at low-redshift; 2) they operate at energies above $15 \mathrm{keV}$, hence allowing detecting photons with enough power to efficiently pierce even through the Compton-thick torus of AGN. Estimates based on observations with PDS on board the BeppoSAX satellite predict that Compton-thick AGN should dominate over unabsorbed AGN in the local universe playing an important role in reproducing the shape and intensity of the cosmic X-ray background (CXB). However coded-mask detectors suffer from heavy systematic effects preventing them from reaching their theoretical limiting sensitivity. We overcome this limit with a new and alternative approach, which has been designed ad hoc to improve the sensitivity of hard X-ray surveys by using IBIS/ISGRI and BAT. Both telescopes are so close in design that their observations can be combined to obtain a more sensitive survey. The observations are combined with resampling, merging, and cross-calibration techniques. We are able to sample limiting fluxes of the order of $\sim 3.3$ times $10^{-12} \mathrm{erg} \mathrm{cm}^{-2} \mathrm{~s}^{-1}$ in the 18 $55 \mathrm{keV}$ energy range. This is called the SIX survey, that stands for Swift-INTEGRAL X-ray survey. The SIX survey extends over a wide sky area of $6200 \mathrm{deg}^{2}$ and it is used to obtain a persistent sample of faint AGN. The source number density $(\log N-\log \mathrm{S}$ ) is a factor of 3 better than current parent surveys of BAT and IBIS/ISGRI alone. I will present a study of the evolution of AGN in the local universe discussing the X-ray luminosity function. The properties of the AGN circum-nuclear environment will be discussed checking the consistency with the AGN unification scheme. Also I will compare the results from the SIX survey to the results predicted for the NuSTAR survey. Finally preliminary results of the all-sky SIX survey are presented.
\end{abstract}

